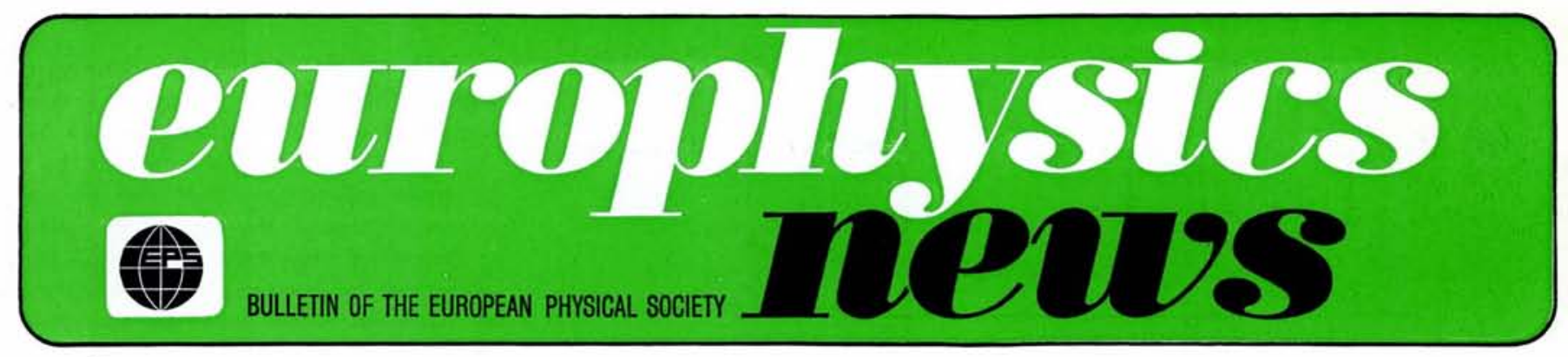

\title{
The Discovery of Tunnelling Supercurrents
}

\author{
Nobel Lecture, 12 December 1973
}

\section{B.D. Josephson, Cambridge}

The events leading to the discovery of tunnelling supercurrents took place while I was working as a research student at the Royal Society Mond Laboratory, Cambridge, under the supervision of Professor Brian Pippard. During my second year as a research student, in 1961-2, we were fortunate to have as a visitor to the laboratory Professor Phil Anderson, who has made numerous contributions to the subject of tunnelling supercurrents, including a number of unpublished results derived independently of myself. His lecture course in Cambridge

$$
\mathrm{H}=-\underset{\mathrm{k}}{2 \Sigma}\left(\varepsilon_{\mathrm{k}}-\mu^{\prime} \mathrm{s}_{\mathrm{kz}}-\sum_{\mathrm{k} \neq \mathrm{k}^{\prime}}^{\Sigma} \mathrm{V}_{\mathrm{k} \mathrm{k}^{\prime}}\left(\mathrm{s}_{\mathrm{kx}} \mathrm{s}_{\mathrm{k}^{\prime} \mathrm{x}}+\mathrm{s}_{\mathrm{ky}} \mathrm{s}_{\mathrm{k}^{\prime} \mathrm{y}}\right),\right.
$$

the first term being the kinetic energy and the second term the interaction energy. In this equation $s_{k x}, s_{k y}$ and $s_{k z}$ are the three components of the $k^{\text {th }}$

$$
\underset{\sim \mathrm{k}}{\mathrm{H}}=2\left(\varepsilon_{\mathrm{k}}-\mu\right) \underset{\sim}{\hat{z}}+2 \underset{\mathrm{k} \neq \mathrm{k}^{\prime}}{\sum} \mathrm{V}_{\mathrm{kk}^{\prime}}{\underset{\sim}{\mathrm{k}^{\prime}} \perp}
$$

where $\hat{z}$ is a unit vector in the $z$ direction and $\perp$ indicates the component of the pseudospin in the $x y$ plane.

One possible configuration of pseudospins consistent with (2) is shown in Fig. 1(a). All the pseudospins lie in the positive or negative $z$ direction, and the direction reverses as one goes through the Fermi surface since $\varepsilon_{\mathrm{k}}-\mu$ changes sign there. If the interaction is attractive, however (corresponding to negative $V_{k k}$, ) a configuration of lower energy exists, in which the pseudospins are tilted out of the negative direction into a plane containing the $z$ axis, and the pseudospin direction changes continuously as one goes through the Fermi surface, as in Fig. 1(b).

The ground state of Fig. 1(b) breaks the symmetry of the pseudospin Hamiltonian (1) with respect to rotation introduced the new concept of 'broken symmetry' in superconductors ${ }^{(1)}$, which was already inherent in his 1958 pseuvity theory ${ }^{(2)}$, which I shall now deAs discussed by Cooper in his Nobel lecture last year(3), according to the Bardeen-Cooper-Schrieffer theory there is a strong positive correlation in a superconductor between of equal and opposite momentum and spin. Anderson showed that in the pseudospin, $\varepsilon_{\mathrm{k}}$ is the single-particle kinetic energy, $\mu$ the chemical potential and $V_{k \mathrm{k}}$, the matrix element for the scattering of a pair of electrons

about the $z$ axis, which is itself a consequence of conservation of number of electrons in the original Hamiltonian. Because of this symmetry a degenerate set of ground states exists, in which the pseudospins can lie in any plane through the $z$ axis. The angle $\Phi$ which this plane makes with the Oxz plane will play an important role in what follows. Anderson made the observation that with a suitable interpretation of the Gor'kov theory ${ }^{(4)}, \Phi$ is also the phase of the complex quantity $\mathrm{F}$ which occurs in that theory.

I was fascinated by the idea of broken symmetry, and wondered whether there could be any way of observing it experimentally. The existence of the original symmetry implies that the absolute phase angle $\Phi$ would be unobservable, but the possibility of observing phase diffe- is perfect the system can be represented by a set of interacting 'pseudospins', with one pseudospin for each pair of electron states. The situation in which both states are unoccupied is represented by a pseudospin in the positive $z$ direction, while occupation of both states is represented by a pseudospin in the negative $z$ direction; other pseudospin orientations correspond to a superposition of the two possibilities.

The effective Hamiltonian for the system is given by

of equal and opposite momentum and spin. The $k^{\text {th }}$ pseudospin sees an effective field

rences betweeen the $\mathrm{F}$ functions in two separate superconductors was not ruled out. However, consideration of the number-phase uncertainty relation suggested that the phase difference $\Delta \Phi$ could be observed only if the two superconductors were able to exchange electrons. When I learnt of observations suggesting that a supercurrent could flow through a sufficiently thin normal region bet-

\section{Contents}

The Discovery of Tunnelling Supercurrents . . . . . 1

The University of Zambia . . 5 Letter to the Editor . . . . 6 Society News . . . . . . 7 Meetings . . . . . . . 8 
(a)

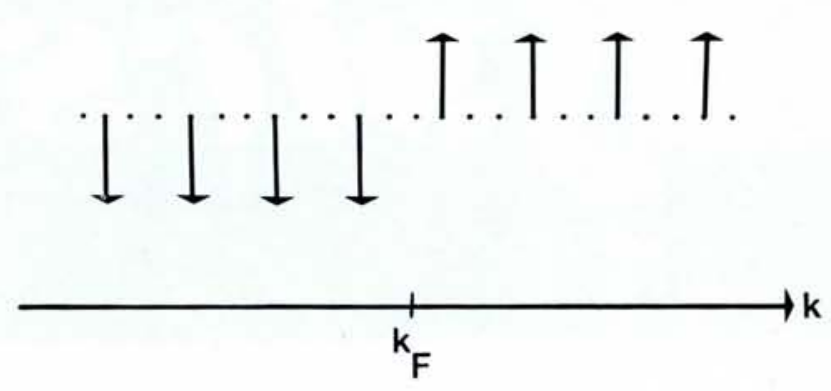

(b)

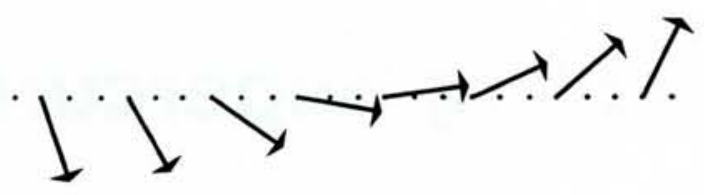

Fig. 1

Pseudospin configurations in (a) a normal metal (b) a superconductor. $k_{F}$ is the Fermi momentum.

ween two superconductors $(5,6)$, I realized that such a supercurrent should be a function of $\Delta \Phi$. I could see in principle how to calculate the supercurrent, but considered the calculation to be too difficult to be worth attempting.

I then learnt of the tunnelling ex-

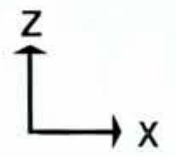

periments of Giaever(7), described in his Nobel lecture(8). Pippard ${ }^{(9)}$ had considered the possibility that a Cooper pair could tunnel through an insulating barrier such as that which Giaever used, but argued that the probability of two electrons tunnelling simultaneously would be very small, so that any effects might be unobservable. This plausible argument is now known not to be valid. However, in view of it I turned my attention to a different possibility, that the normal currents through the barrier might be modified by the phase difference. An argument in favour of the existence of such an effect was the fact that matrix elements for processes in a superconductor are modified from those for the corresponding processes in a normal metal by the so-called coherence factors ${ }^{(3)}$, which are in turn dependent on $\Delta \Phi$ (though the $\mathrm{u}_{\mathrm{k}}$ 's and $v_{k}$ 's of the BCS theory). At this time there was no theory available to calculate the tunnelling current, apart from the heuristic formula of Gia$\operatorname{ever}^{(7)}$, which was in agreement with experiment but could not be derived from basic theory. I was able, however, to make a qualitative prediction concerning the time dependence of the current. Gor'kov(4) had noted that the $\mathrm{F}$ function in his theory should be time-dependent, being proportional to $e^{-2 i \mu t / \hbar}$, where $\mu$ is the chemical potential as before ${ }^{(10)}$. The phase $\Phi$ should thus obey the relation

$$
\partial \Phi / \partial t=-2 \mu / \hbar,
$$

while in a two-superconductor system the phase difference obeys the relation

$$
\frac{\partial}{\partial t}(\Delta \Phi)=2 \mathrm{eV} / \hbar
$$

where $\mathrm{V}$ is the potential difference between the two superconducting regions, so that

$$
\Delta \Phi=2 \mathrm{eVt} / \hbar+\text { const. }
$$

Since nothing changes physically if $\Delta \Phi$ is changed by a multiple of $2 \pi$, I was led to expect a periodically varying current at a frequency $2 \mathrm{eV} / \mathrm{h}$.

The problem of how to calculate the barrier current was resolved when one day Anderson showed me a preprint he had just received from Chicago $^{(11)}$, in which Cohen, Falicov and Phillips calculated the current flowing

$$
I=I_{0}(V)+I_{1}^{\prime}(V) \cos (\Delta \Phi)+I_{1}(V) \sin (\Delta \Phi) .
$$

At finite voltages the linear increase with time of $\Delta \Phi$ implies that the only contribution to the dc current comes from the first term, which is the same as Giaever's prediction, thus extending the results of Cohen et al. to the two-superconductor case. The second term had a form consistent with my expectations of a $\Delta \Phi$ dependence of the current due to tunnelling of quasiparticles. The third term, however, was completely unexpected, as the coefficient $I_{1}(V)$, unlike $I_{0}(V)$ and $I_{1}{ }^{\prime}(V)$, was an even function of $\mathrm{V}$ and would not be expected to vanish when $V$ in a superconductor-barrier-normal metal system, confirming Giaever's formula. They introduced a new and very simple way to calculate the barrier current - they simply used conservation of change to equate it to the time derivative of the amount of charge on one side of the barrier. They evaluated this time derivative by perturbation theory, treating the tun-

was put equal to zero. The $\Delta \Phi$ dependent current at zero voltage had the obvious interpretation of a supercurrent, but in view of the qualitative argument mentioned earlier I had not expected a contribution to appear of the same order of magnitude as the quasi-particle current, and it was some days before I was able to convince myself that I had not made an error in the calculation.

Since $\sin (\Delta \Phi)$ can take any value from -1 to +1 , the theory predicted a value of the critical supercurrent of $I_{1}(0)$. At a finite voltage $V$ an 'ac su- nelling of electrons through the barrier as a perturbation on a system consisting of two isolated subsystems between which tunnelling does not take place.

I immediately set to work to extend the calculation to a situation in which both sides of the barrier were superconducting. The expression obtained was of the form

\section{percurrent' of amplitude}

$$
V\left\{\left[\mathrm{I}_{1}(\mathrm{~V})\right]^{2}+\left[\mathrm{I}_{1}(\mathrm{~V})\right]^{2}\right\}
$$

and frequency $2 \mathrm{eV} / \mathrm{h}$ was expected. As mentioned earlier, the only contribution to the dc current in this situation $(V \neq 0)$ comes from the $I_{0}(V)$ term, so that a two-section currentvoltage relation of the form indicated in Fig. 2 is expected.

I next considered the effect of superimposing an oscillatory voltage at frequency $v$ on to a steady voltage $\mathrm{V}$. By assuming the effect of the oscillatory voltage to be to modulate the 
frequency of the ac supercurrent I concluded that constant-voltage steps would appear at voltages $V$ for which the frequency of the unmodulated ac supercurrent was an integral multiple of $v$, i.e. when $V=n h v / 2 e$ for some integer $\mathrm{n}$.

The embarrassing feature of the theory at this point was that the effects predicted were too large! The magnitude of the predicted supercurrent was proportional to the normal state conductivity of the barrier, and of the same order of magnitude as the jump in current occuring as the voltage passes through that at which production of pairs of quasiparticles becomes possible. Examination of the literature showed that possibly dc supercurrents of this magnitude had been observed, for example in the first published observation of tunnelling between two evaporated-film superconductors by $\mathrm{Ni}$ col, Shapiro and Smith(12) (Fig. 3). Giaever ${ }^{(13)}$ had made a similar observation, but ascribed the supercurrents seen to conduction through metallic shorts through the barrier layer. As supercurrents were not always seen, it seemed that the explanation in terms of shorts might be the correct one, and the whole theory might have been of mathematical interest only (as was indeed suggested in the literature soon after).

Then a few days later Phil Anderson walked in with an explanation for the missing supercurrents, which was sufficiently convincing for me to decide to go ahead and publish my cal-

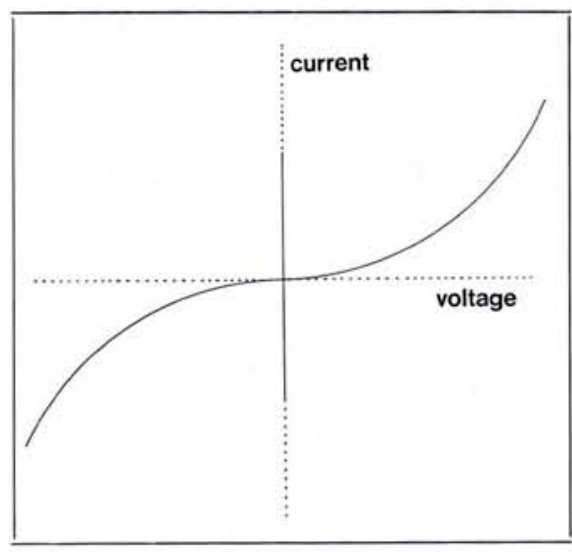

Fig. 2.

Predicted two-part current-voltage characteristic of a superconducting tunnel junction.

culation $^{(14)}$, although it turned out later not to have been the correct explanation. He pointed out that my relation between the critical supercurrent and the normal state resistivity depended on the assumption of time-reversal symmetry, which would be violated if a magnetic field were present. I was able to calculate the magnitude of the effect by using the Ginzburg-Landau theory to find the effect of the field on the phase of the $F$ functions, and concluded that the Earth's field could have a drastic effect on the supercurrents.

Brian Pippard then suggested that I should try to observe tunnelling supercurrents myself, by measuring the characteristics of a junction in a compensated field. The result was negative - a current less than a thousandth of the predicted critical current was sufficient to produce a

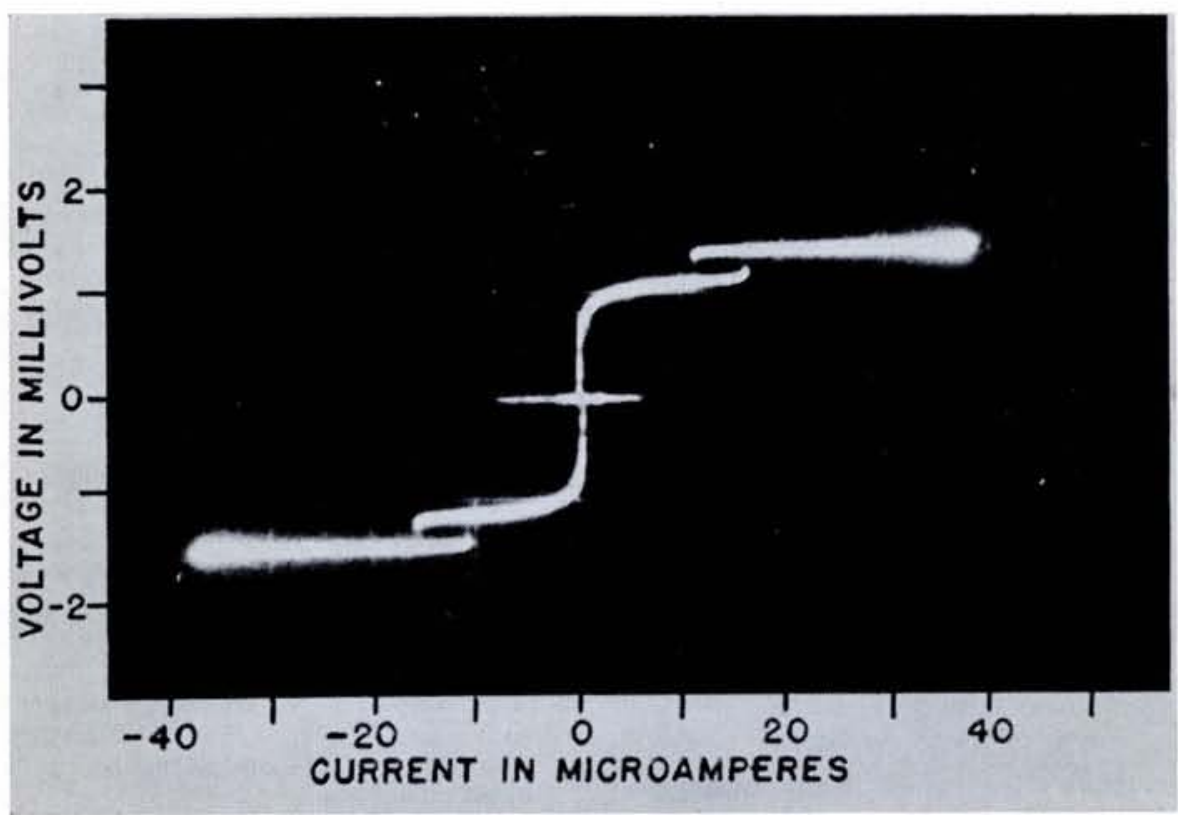

Fig. 3

The first published observation of tunnelling between two evaporated-film superconductors (Nicol, Shapiro and Smith, reference 6). A zero-voltage supercurrent is clearly visible. It was not until the experiments of Anderson and Rowell (reference 15) that such supercurrents could be definitely ascribed to the tunnelling process.

\section{Defects in Crystalline Solids}

Editors : S. AMELINCKX R. GEVERS, J. NIHOUL

Vol. 8 : Ion Implantation

By G. DEARNALEY, et al.,

Atomic Energy Research

Establishment, Harwell, England.

1973. 818 pages.

Dfl. 225.00 (about US \$ 81.80 )

Vol. 7 : Computed Electron

Micrographs and Defect

Identification

By A.K. HEAD et al.,

University of Melbourne, Australia.

1973. 410 pages.

Dfl. 100.00 (about US $\$ 36.40$ )

Vol. 6 : Irradiation Effects

in Fissile Materials

By J. LETEURTRE and Y. QUÉRE,

Centre d'Etudes Nucléaires,

Fontenay-aux-Roses, France.

1972. 138 pages.

Dfl. 45.00 (about US $\$ 16.40$ )

Vol. 5 : Internal Friction

of Structural Defects

in Crystalline Solids

By R. DE BATIST,

Studiecentrum voor Kernenergie,

Mol and University of Antwerp,

Belgium.

1972. 490 pages.

Dfl. 120.00 (about US \$43.60)

Vol. 4 : The Electronic Structure of Point Defects

As Determined by Mössbauer

Spectroscopy and by Spin Resonance

By G.K. WERTHEIM, Bell Telephone

Laboratories, Murray Hill, N.J., U.S.A.,

A. HAUSMANN and W. SANDER,

Institute of Physics, Technical

University of Rhine/Westphalia,

Aachen, Germany.

1971. 234 pages.

Dfl. 65.00 (about US \$23.60)

Vol. 3 : Quench Hardening in Metals

By H. KIMURA, Tohoku University,

Sendai, Japan, and R. MADDIN,

University of Pennsylvania,

Philadelphia, Pa., U.S.A.

1971. 136 pages.

Dfl. 36.00 (about US \$13.10)

Vol. 2 : Field-Ion Microscopy

By K.M. BOWKETT, University of

Cambridge Metallurgy Department,

Fellow of Christ's College,

Cambridge, and D.A. SMITH,

University of Oxford Metallurgy

Department, Fellow of Trinity

College, Oxford.

1970. 268 pages.

DfI. 54.00 (about US \$19.60)

Vol. 1 : The Observation of Atomic Collisions in Crystalline Solids

By R.S. NELSON, Atomic Energy

Research Establishment,

Harwell, England.

1968. 296 pages.

DfI. 50.00 (about US $\$ 18.20$ )

\section{NORTH-HOLLAND}

P.O. Box 211, Amsterdam, The Netherlands Distributed in the United States and Canada by American Elsevier Publishing New York N.Y., 52 Vanderbilt Avenue. 
detectable voltage across the junction. This experiment was at one time to be written up in a chapter of my thesis entitled 'Two Unsuccessful Experiments in Electron Tunnelling between Superconductors'.

Eventually Anderson realized that the reason for the non-observation of dc supercurrents in some specimens was that electrical noise transmitted down the measuring leads to the specimen could be sufficient in high-resistance specimens to produce a current exceeding the critical current. Together with John Rowell he made some low resistance specimens and soon obtained convincing evidence ${ }^{(15)}$ for the existence of tunnelling supercurrents, shown particularly by the sensitivity to magnetic fields, which would not be present in the case of conduction through a metallic short. In one specimen they found a critical current of $0.30 \mathrm{~mA}$ in the Earth's magnetic field. When the field was compensated, the critical current increased by more than a factor of two, to $0.65 \mathrm{~mA}$, while a field of $2 \mathrm{mT}$ was sufficient to destroy the zero-voltage supercurrents completely. Later Rowell(16) investigated the field dependence of the critical current in detail, and obtained results related to the diffraction pattern of a single slit, a connection first suggested by J.C. Phillips (unpublished). This work was advanced by Jaklevic, Lambe, Silver and Mercereau(17), who connected two junctions in parallel and were able to observe the analogue of the Young's slit interference experiment. The sensitivity of the critical current to applied magnetic field can be increased by increasing the area enclosed between the two branches of the circuit, and Zimmerman and Silver ${ }^{(18)}$ were able to achieve a sensitivity of $10^{-13} \mathrm{~T}$.

Indirect evidence for the ac supercurrents came soon afier. Shapiro(19) shone microwaves on to a junction and observed the predicted appearance of steps in the current-voltage characteristics. The voltages at which the steps occurred changed as the frequency of the microwaves was changed, in the manner expected. In 1966, Langenberg, Parker and TayIor ${ }^{(20)}$ measured the ratio of voltage to frequency to 60 parts per million and found agreement with the value of $\mathrm{h} / 2 \mathrm{e}$ then accepted. Later they increased their accuracy sufficiently to be able to discover errors in the previously accepted values of the fundamental constants and derive more accurate estimates ${ }^{(21,22)}$, thus carrying out to fruition an early suggestion of
Pippard (unpublished). The ac supercurrent is now used to compare voltages in different standards laboratories without the necessity for the interchange of banks of standard cells. If two laboratories irradiate specimens with radiation of the same frequency, constant-voltage steps appear at identical voltages. The intercomparison of frequencies can be carried out in a straightforward manner by transmission of radio signals.

At the end of 1963, the evidence for the existence of the ac supercurrent was only indirect. John Adkins and I tried to observe the effect by coupling together two junctions by a short $(\sim 0.2 \mathrm{~mm})$ thin-film transmission line. The idea was that radiation emitted by one junction would modify the charecteristics of the other. The experiment, planned to form the second part of the thesis chapter referred to above, was unsuccessful, for reasons which are still unclear. Later, Giaever ${ }^{(23)}$ was able to observe the ac supercurrent by a similar method to the one we had considered, and then Yanson, Svistunov and Dmitrenko(24) succeeded in observing radiation emitted by the ac supercurrent with a conventional detector.

Finally, I should like to describe the SLUG ${ }^{(25)}$, developed in the Royal Society Mond Laboratory by John Clarke while he was a research student. John was attempting to make a highsensitivity galvanometer using the previously described magnetic interferometers with two junctions connected in parallel. One day Paul Wraight, who shared a room with John, observed that the fact that one cannot solder niobium using ordinary solder must mean that if one allows a molten blob of solder to solidify in contact with niobium there must be an intermediate layer of oxide, which might have a suitable thickness to act as a tunnelling barrier. This proved to be the case. However, in John's specimens, in which a niobium wire was completely surrounded by a blob of solder, the critical current through the barrier proved to be completely insensitive to externally applied magnetic fields. It was, however, found to be sensitive to the magnetic field produced by passing a current through the central wire. This fact led to the development of a galvanometer with sensitivity of $10^{-14}$ volts at a time constant of $1 \mathrm{sec}$.

There have been many other developments which I have not had time to describe here. I should like to conclude by saying how fascinating it has been for me to watch over the years the many developments in laboratories over the world, which followed from asking one simple question, namely what is the physical significance of broken symmetry in superconductors?

REFERENCES AND FOOTNOTES

1. P. W. Anderson, Concepts in Solids, W. A. Benjamin, Inc., New York, 1963.

2. P. W. Anderson, Phys. Rev. 112, 1900 (1958).

3. L. N. Cooper, Les Prix Nobel en 1972, Nobel Foundation 1973 , p. 64.

4. L. P. Gor'kov, J. Exptl. Theoret. Phys. (URSS) 36, 1918 (1959) ; translation: Soviet Phys. JETP 9, 1364 (1959).

5. H. Meissner, Phys. Rev. 117, 672 (1960).

6. P. H. Smith, S. Shapiro, J. L. Miles and J. Nicol, Phys. Rev. Letters 6, 686 (1961).

7. I. Giaever, Phys. Rev. Letters 5, 464 (1960).

8. I. Giaever, Les Prix Nobel en 1973, Nobel Foundation 1974, p.

9. A. B. Pippard, Proceedings of the VIIth International Conference on Low Temperature Physics, ed. G. M. Graham and A. C. Hollis Hallett, North-Holland, Amsterdam 1961, p. 320 .

10. Gor'kov's result may be extended to finite temperatures by the following argument. The density operator of a system in equilibrium has the form $\mathrm{Z}^{-1} \exp \{-\beta(\mathrm{H}-\mu \mathrm{N})$, where $\mathrm{H}$ must contain a small symmetry-breaking term in order to set up an ensemble in which $\Phi$ has a definite value. Since this operator commutes with $\mathrm{H}-\mu \mathrm{N}$, all quantities are timeindependent if the Hamiltonian of the system is taken to be $\mathrm{H}-\mu \mathrm{N}$. Transition to a situation where the time dependence is given by the true Hamiltonian $\mathrm{H}$ can be accomplished by means of a gauge transformation, and consideration of the effect of this transformation on the electron operators gives immediately Gor'kov's result $F \propto \exp (-2 i \mu t / h)$.

11. M. H. Cohen, L. M. Falicov and J. C. Phillips Phys. Rev. Letters 8, 316 (1962).

12. J. Nicol, S. Shapiro and P. H. Smith, Phys. Rev. Letters 5, 461 (1960).

13. I. Giaever, Phys. Rev. Letters 5, 464 (1960).

14. B. D. Josephson, Phys. Letters 1, 251 (1962).

15. P. W. Anderson and J. M. Rowell, Phys. Rev. Letters 10, 230 (1963).

16. J. M. Rowell, Phys. Rev. Letters 11, 200 (1963)

17. R. C. Jaklevic, J. Lambe, A. H. Silver and J. E. Mercereau, Phys. Rev. Letters 12, 159 (1964).

18. J. E. Zimmerman and A. H. Silver, Phys. Rev. 141, 367 (1966).

19. S. Shapiro, Phys. Rev. Letters 11, 80 (1963).

20. D. N. Langenberg, W. H. Parker and B. N. Taylor, Phys. Rev. 150, 186 (1966).

21. W. H. Parker, B. N. Taylor and D.N. Langenberg, Phys. Rev. Letters 18, 287 (1967).

22. B. N. Taylor, W. H. Parker and D. N. Langenberg. The Fundamental Constants and Quantum Electrodynamics, Academic Press, New York and London, 1969.

23. I. Giaever, Phys. Rev. Letters 14, 904 (1965)

24. I. K. Yanson, V. M. Svistunov and I. M. Dmitrenko, J. Exptl. Theoret. Phys. (URSS) 21. 650 (1965); translation: Soviet Phys. JETP 48, 976 (1965).

25. J. Clarke, Phil. Mag. 13, 115 (1966). 
As reply to the letter by F. Janouch, Prague concerning "Unemployed physicists to assist developing countries?" we received the article given below.

\section{Introduction}

The University of Zambia was established in 1965, following the Report of an Anglo-American team headed by Sir John Lockwood, which visited the country in 1963. The University is dedicated to the task of responding to the real needs of Zambia. At the same time, as a member of the Association of Commonwealth Universities, it is determined to earn the respect and proper recognition of the international university community.

The University campus is situated on the outskirts of Lusaka. The Physics Building, which was occupied in 1968, presently houses Physics, Mathematics, the Computer and Data Processing Centre, the office of the Dean of the School of Natural Sciences, and small machine-tool and electronics workshops. As further buildings come to operation Physics will take over more of the Physics Building - there is ample room for expansion for many years to come.

\section{Degree/Courses}

Entrance to the University is at GCE ' $O$ ' Level, and the duration of the degree course for the B.Sc. degree or the B.Sc. (ed.) is four years. Degrees are offered in Physical sciences and in Physics with mathematics ; it is hoped to offer a degree in Physics in the future. One year of Physics is also taken by students studying engineering, medicine, agriculture, or the biological sciences. The major part of the teaching effort in Physics is therefore at introductory level; the main 1st-year course for 280 students is being developed on a self-paced modular basis, and we would particularly welcome colleagues with experience in 'Keller-plan' or similar mastery-learning introductory courses. Limited opportunities also exist for teaching courses in geophysics and materials science, to both engineers and scientists, and for developing and teaching 'Applied Physics' courses.

\section{Teaching and Research}

The University Council regards twelve formal contact hours a week (32 weeks p.a.) as a fair teaching load. This leaves adequate time for staff

\section{The University of Zambia}

\section{A. H. Ward, Lusaka}

members to pursue their own research work. Financial provision for research has been very generous, by African standards, and the laboratories are well equipped. In addition to physics equipment, the University possesses an IBM 1130 computer, electron microscope and electron diffraction camera, a range of materials-science and metal-physics and geophysics research equipment, and houses $\mathrm{nmr}$ and Packard Tricarb-Autogamma spectrometers and a 400-channel Laben spectroscope.

In physics, metal-physics research developed well in 1967-1968 and a request to the RCM (RST) group of mining companies in 1968 for substantial support was successful. An RCM Research Fellow was appointed in 1969, and further equipment purchased. The three small research laboratories now house an Instron TM tensile testing machine, Philips $\mathrm{X}$-ray generator with powder cameras and Universal flat-plate camera, micro-densitometer, Servomet spark machiner, 4" electromagnet, Pye precision decade potentiometer, Hille rolling mill, Zeiss metallurgical microscope, and a range of furnaces, polishing, and ancillary equipment. In addition, RCM provides technical support in its own 'Copperbelt' laboratories, e.g. with metal working and analysis. Investigations include the recrystallisation of wirebar copper, an assessment of the spring-elongation test currently used in copper quality control, work on the theories of recrystallisation and of the annealing behaviour of the point defects in metals; two important research papers have been published.

This successful metal-physics work is being expanded with a 2nd Research Fellow and with further backing from industry and mines to a broader 'materials-science' research effort, including ceramics and refractories; new colleagues with relevant interest would be particularly welcome.

The geophysics research team started in 1970 and has support from NCCM (AAC) and RCM groups of mining companies and from other outside firms and departments. It has made a strong start in four fields seismic and seismic-refraction, geothermal, gravitational and geoelectrical research. It is working in close cooperation with professional colleagues in Government departments and the mining companies. There are two small research labs, the seismological observatory, and a geophysics Land-

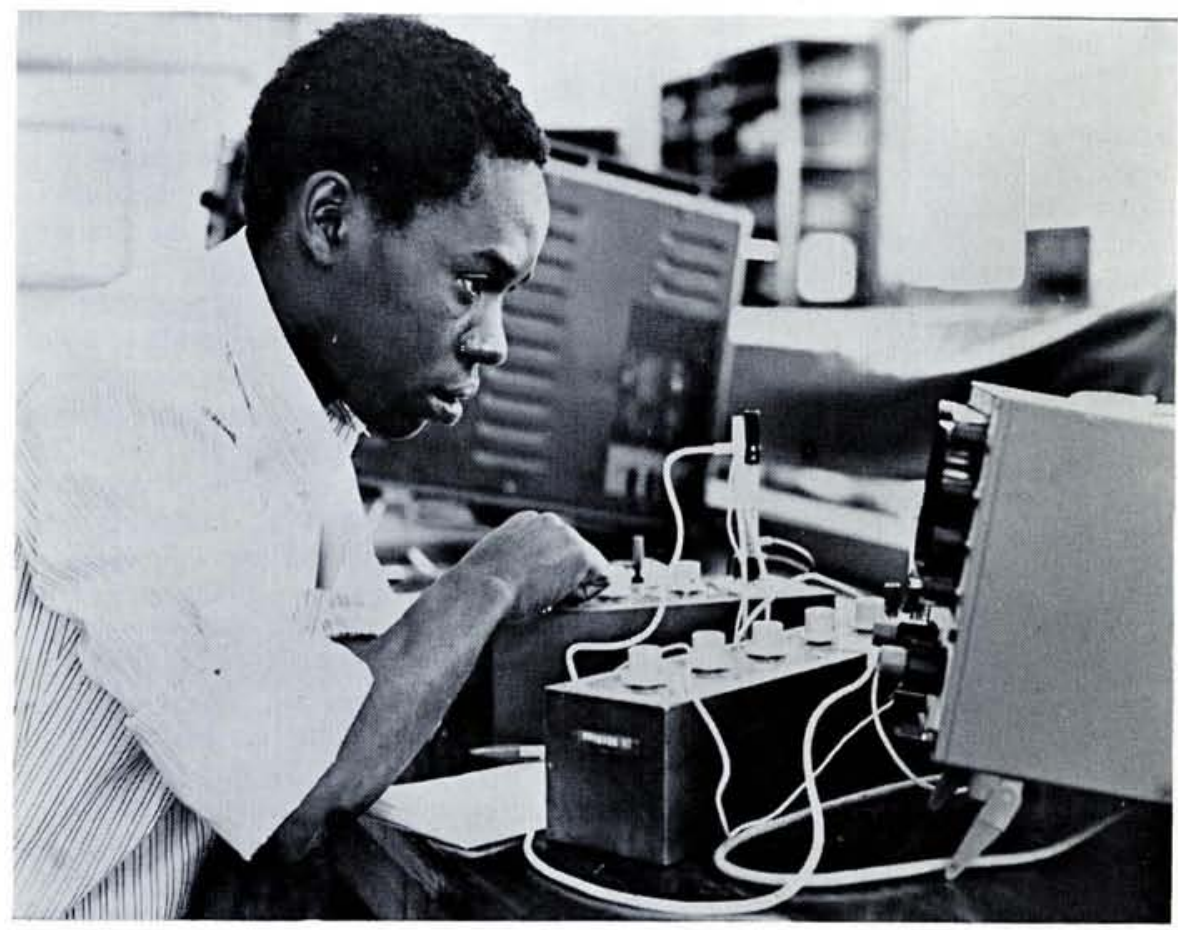

University of Zambia, Lusaka - Second year student, A. Muneka on electronic equipment. 